\title{
Multi-response central composite design of the mineralization and removal of aniline by subcritical water oxidation method
}

\author{
Erdal Yabalak ${ }^{1}$ (D) $\cdot$ İpek Topaloğlu ${ }^{1}$ Ahmet Murat Gizir ${ }^{1}$
}

Received: 20 August 2018 / Accepted: 18 February 2019 / Published online: 26 February 2019

(c) The Author(s) 2019

\begin{abstract}
Aniline is used by many industrial organizations and is released into the environment in high amounts and pollutes the water. Due to the damages to human health, there is a need for effective and environmentally friendly methods to remove this compound from the water. The aim of this work is to mineralize and remove aniline by investigating total organic carbon removal and aniline removal, respectively, via using the eco-friendly method, subcritical water oxidation and green oxidizing agent, $\mathrm{H}_{2} \mathrm{O}_{2}$. The effect of process variables such as temperature, treatment time and $\mathrm{H}_{2} \mathrm{O}_{2}$ concentration were evaluated. Total organic carbon removal of aniline was obtained as $92.73 \%$ in $80 \mathrm{~min}$ of treatment time and in the presence of $60 \mathrm{mM}$ of $\mathrm{H}_{2} \mathrm{O}_{2}$ at $403 \mathrm{~K} .99 .21 \%$ of aniline removal was achieved based on the UV spectrophotometric analysis at these conditions. The response surface method was used to optimize the performed model and the effect of the above-mentioned parameters. The reliability of the method was provided by ANOVA.
\end{abstract}

Keywords Aniline $\cdot$ Response surface methodology $\cdot$ Mineralization $\cdot$ TOC removal $\cdot$ Subcritical water · Oxidation · Degradation

\section{Introduction}

Aniline and its derivatives are important for the petrochemical, pharmaceutical and textile industries and they are also used in dye production. It is estimated that the release of aniline to the environment is 30,000 tonnes per annum due to the illegal release of municipal and industrial wastewater and overuse of pesticides [1]. Aniline is one of the 129 priority pollutants, identified by the EPA, due to being a toxic nonirreversible material with low biodegradability [2].

Several oxidation techniques such as ozonation [3], photochemical [4], electrochemical [5], and photocatalysis [6], have been carried out to remove aniline from water. Various oxidants $\left(\mathrm{O}_{2}, \mathrm{H}_{2} \mathrm{O}_{2}\right.$, and $\left.\mathrm{O}_{3}\right)$ were used in catalysed or non-catalysed media and various amounts of TOC and COD removal were observed in these studies [7, 8]. Furthermore, many methods such as oxidation [9], extraction [10], biodegradation [11], and adsorption [12] were performed to

Erdal Yabalak

yabalakerdal@gmail.com

1 Department of Chemistry, Faculty of Arts and Science, Mersin University, Çiftlikköy Campus, 33343 Mersin, Turkey degrade aniline or decontaminate aniline-polluted water. However, most of the methods fail to provide the desired yield or they are not satisfactory in the major issues, such as treatment time, operating cost, or environmental awareness.

Herein, subcritical water oxidation (SWO), which is an effective and environmentally friendly alternative to these methods, can overcome the above-mentioned problems. Subcritical water has some characteristic physicochemical properties such as low dielectric constant, low surface tension, low viscosity, average polarity and suitable solvent properties. It is defined as water at a high temperature of 373-647 K and high pressure that is necessary to keep the water in the liquid state at the related temperature [13].

SWO is a thermochemical process, similar to the wet air oxidation process, in which hydroxyl radicals and active oxygen species are formed at high temperatures and pressures [14]. It depends on the oxidation of organic compounds in the aqueous phase at a high temperature (373-647 K) and pressure. In this way, it is possible to convert harmful organic compounds and micro-pollutants into harmless organic compounds such as $\mathrm{CO}_{2}$ and $\mathrm{H}_{2} \mathrm{O}$. SWO is generally used for purification of water-containing toxic organic wastes. Complex organic compounds are converted into simpler biodegradable species by SWO. In this type of oxidation 
process, a large majority of organic content is stoichiometrically oxidized. Namely, carbon is oxidized to carbon dioxide, hydrogen is oxidized to water, halogens are oxidized to halides, sulphides are oxidized to sulphates, phosphorus is oxidized to phosphates and nitrogen is oxidized to ammonia, or elemental nitrogen [15]. The compounds with long-chain structure are converted into various intermediates, but these intermediates are generally not stable and are then oxidized to the final products, $\mathrm{CO}_{2}$ and $\mathrm{H}_{2} \mathrm{O}$ [16]. SWO process, in which $\mathrm{H}_{2} \mathrm{O}_{2}$ is used as an oxidizer, is effective in the application of wastewater-containing medium and high concentrations of organic carbon. Furthermore, $\mathrm{H}_{2} \mathrm{O}_{2}$ is an ecological oxidant that is not toxic and does not cause any harmful by-products [17].

The experimental parameters (temperature, time, oxidant concentration) of the process and the optimization of the process were performed using the response surface method (RSM) which consists of a series of mathematical and statistical techniques. RSM is used to exhibit the effect of experimental values of any factors on the response to find the optimum process conditions and the most effective variables on the response. Also, it is used to define the relationship between response and independent variables, whereas univariate method, namely the one-factor-at-a-time analysis, allows to optimize methods by following one factor at a time where keeping other factors constant [18]. Fortunately, RSM provides to evaluate the performance of a system requiring a limited number of experiments [19]. These techniques investigate the effects of the independent variables (factors) on the response alone or all factors together. In this manner, the central composite design (CCD), which is one of the most preferred RSM designs, can meet expectations [19].

In this study, SWO, which has been used in the degradation of many pollutants, was used along with the oxidizing agent, $\mathrm{H}_{2} \mathrm{O}_{2}$. Mineralization and removal percentage of aniline were, respectively, followed by the total organic carbon (TOC) analysis and the UV spectrophotometric analysis of the residual. TOC measurement is the best-known method for determining the organic content of a water sample. The amount of carbon dioxide produced from the total organic carbon content of a sample gives a TOC value [20]. Besides, the UV spectrophotometric analysis is a practical way to follow the removal of a UV-active pollutant from an aqueous solution [21]. CCD was applied to this work to optimize the process variables and evaluate mineralization and removal condition for maximizing the mineralization and removal percentage.

\section{Experimental}

\section{Materials}

Aniline and $\mathrm{H}_{2} \mathrm{O}_{2}$ were purchased from Merck (Germany), $\mathrm{N}_{2}$ gas was provided from Linde Gas (Turkey). Ultrapure water was prepared using the Millipore Milli-Q Advantage A10, Merck (Germany). A home-made stainless steel reactor used in the previous work was used for degradation experiments [22]. UV analyses were recorded by the Shimadzu UV-1601 (Germany) spectrometer.

\section{Methods}

\section{Degradation method}

The aqueous solution of aniline (100 ppm) was prepared by dissolving a certain volume in deionized water. $150 \mathrm{~mL}$ of this solution was put into the reactor following a certain amount of oxidizing agent, $\mathrm{H}_{2} \mathrm{O}_{2}$. The inner pressure of the reactor was provided by $\mathrm{N}_{2}$ gas. Degradation experiments were carried out at 5 different temperatures $(352.55 \mathrm{~K}$, $373 \mathrm{~K}, 403 \mathrm{~K}, 433 \mathrm{~K}$ and $453.45 \mathrm{~K}$ ), 5 different concentrations $(12.73 \mathrm{mM}, 40 \mathrm{mM}, 80 \mathrm{mM}, 120 \mathrm{mM}$ and $147.27 \mathrm{mM})$ and 5 different treatment times $(9.55 \mathrm{~min}, 30 \mathrm{~min}, 60 \mathrm{~min}$, $90 \mathrm{~min}$ and $120 \mathrm{~min}$ ) as shown in Table $1.25 \mathrm{~mL}$ of each treated sample was kept for TOC and UV analysis.

\section{TOC analysis}

TOC analyses were performed using the TOC-L CPN analyser (Shimadzu, Japan) with ASI-L injection port. The total organic carbon removals were measured in $25 \mathrm{~mL}$ TOC test sample vials. The five-point calibration curve was used to determine the TOC values of the samples. TOC removal percentages were calculated according to the equation that was given in the previous work [19].
Table 1 Experimental design of independent variables employing in the CCD

\begin{tabular}{llcrrrr}
\hline Independent variables & Factors & \multicolumn{2}{l}{ Intervals and levels } \\
\cline { 3 - 7 } & & \multicolumn{1}{c}{$-\alpha$} & \multicolumn{1}{c}{-1} & \multicolumn{1}{c}{0} & \multicolumn{1}{c}{1} \\
\hline Temperature $(\mathrm{K})$ & $x_{1}$ & 352.55 & 373 & 403 & 433 & 453.45 \\
$\mathrm{H}_{2} \mathrm{O}_{2}$ concentration $(\mathrm{mM})$ & $x_{2}$ & 12.7 & 40 & 80 & 120 & 147 \\
Treatment time $(t)$ & $x_{3}$ & 9.54 & 30 & 60 & 90 & 110 \\
\hline
\end{tabular}

$\alpha=1.682$ 


\section{UV-Vis analysis}

The removal of aniline (aniline removal) was spectrophotometrically determined using the UV spectrophotometer. The percentage yield of aniline removal of each experiment was determined via comparing the absorbance $(254 \mathrm{~nm}$ ) of the stock solution and treated samples according to the equation which was given in the previous work [19]. However, it was obtained that $\mathrm{H}_{2} \mathrm{O}_{2}$ absorbs the same wavelength. Thus, unreacted $\mathrm{H}_{2} \mathrm{O}_{2}$, which may have remained after treatment process, was removed by treating samples with $\mathrm{NaBiO}_{3}$ for $2 \mathrm{~h}$. Samples were analysed by the UV-Vis spectroscopy after filtering the samples through $0.45-\mu \mathrm{m}$ PTFE syringe filters.

\section{CCD modelling}

A CCD model is a full or fractional factorial quadratic design which consists of factorial, center, and axial points situated in the experimental region $[19,23]$. The multi-response CCD method was applied to evaluate the optimum experimental conditions of aniline degradation. The responses of TOC removal and aniline removal were functioned as responses. The interactions of temperature, oxidant concentration and treatment time were assessed and the effects of these factors on the responses were analysed. Design Expert 9.0.6.2 was used to analyse the experimental results and to obtain a regression model. The list of 20 experimental runs and their experimental and predicted results are given in Table 2. TOC removal and aniline removal percentages of treated samples were calculated using the equation given in the previous work [19]. Obtained results were statistically evaluated by variance analysis (ANOVA) to analyse the proficiency of the proposed model for aniline degradation.

\section{Results and discussion}

Experimental results of TOC removal and aniline removal percentages were demonstrated in Table 2 along with their predicted values. The highest experimental and predicted TOC removal percentages were obtained as $92.73 \%$ and $93.37 \%$, respectively, at runs 17 and 12 . The lowest values for experimental and predicted TOC removal percentages were obtained as $34.60 \%$ and $44.40 \%$ at run 3 . However, aniline removal percentages were obtained to be higher than TOC removal percentages in both cases of experimental and predicted values. Aniline removal was obtained with the highest values of $99.21 \%$ and $100.48 \%$, respectively, at runs 11 and 12 . The lowest experimental and predicted aniline removal percentages were obtained as $63.64 \%$ and $63.90 \%$, respectively, at runs 15 and 18 , respectively. As the above-mentioned data show, maximum and minimum TOC and aniline removal values were obtained at different
Table 2 Experimental and predicted results of TOC and aniline removal percentages

\begin{tabular}{|c|c|c|c|c|c|c|c|c|c|c|c|}
\hline \multirow[t]{2}{*}{ Run } & \multirow[t]{2}{*}{$x_{1}$} & \multirow[t]{2}{*}{$x_{2}$} & \multirow[t]{2}{*}{$x_{3}$} & \multicolumn{4}{|c|}{ TOC removal, \% } & \multicolumn{4}{|c|}{ Aniline removal. \% } \\
\hline & & & & Exp. & CCD pre. & Residual & $\mathrm{AD}, \%$ & Exp. & CCD pre. & Residual & $\mathrm{AD}, \%$ \\
\hline 1 & 453.45 & 80 & 60 & 69.78 & 65.65 & 4.130 & 5.92 & 83.41 & 83.75 & 0.340 & 0.41 \\
\hline 2 & 403 & 12.73 & 60 & 49.57 & 56.00 & 6.430 & 12.97 & 70.48 & 72.84 & 2.360 & 3.35 \\
\hline . & 352.55 & 80 & 60 & 34.60 & 44.40 & 9.800 & 28.32 & 70.51 & 74.15 & 3.640 & 5.16 \\
\hline 4 & 403 & 147.27 & 60 & 91.79 & 91.02 & 0.770 & 0.84 & 96.60 & 98.23 & 1.630 & 1.69 \\
\hline 5 & 403 & 80 & 60 & 92.46 & 87.64 & 4.820 & 5.21 & 82.05 & 95.05 & 13.000 & 15.84 \\
\hline 6 & 433 & 120 & 30 & 84.91 & 87.02 & 2.110 & 2.48 & 97.92 & 97.70 & 0.220 & 0.22 \\
\hline 7 & 433 & 40 & 90 & 85.84 & 83.20 & 2.640 & 3.08 & 96.94 & 94.87 & 2.070 & 2.14 \\
\hline 8 & 403 & 80 & 60 & 84.19 & 87.64 & 3.450 & 4.10 & 97.41 & 95.05 & 2.360 & 2.42 \\
\hline 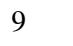 & 373 & 40 & 90 & 69.53 & 63.42 & 6.110 & 8.79 & 89.47 & 86.87 & 2.600 & 2.91 \\
\hline 10 & 403 & 80 & 9.55 & 75.99 & 76.78 & 0.790 & 1.04 & 83.57 & 83.72 & 0.150 & 0.18 \\
\hline 11 & 403 & 80 & 60 & 80.39 & 87.64 & 7.250 & 9.02 & 99.21 & 95.05 & 4.160 & 4.19 \\
\hline 12 & 403 & 80 & 110.45 & 88.50 & 93.37 & 4.870 & 5.50 & 96.65 & 100.48 & 3.830 & 3.96 \\
\hline 13 & 433 & 120 & 90 & 84.81 & 87.58 & 2.770 & 3.27 & 95.57 & 94.67 & 0.900 & 0.94 \\
\hline 14 & 373 & 120 & 90 & 71.82 & 66.35 & 5.470 & 7.62 & 86.49 & 83.34 & 3.150 & 3.64 \\
\hline 15 & 433 & 40 & 30 & 46.83 & 48.29 & 1.460 & 3.12 & 63.64 & 63.98 & 0.340 & 0.53 \\
\hline 16 & 403 & 80 & 60 & 84.99 & 87.64 & 2.650 & 3.12 & 95.85 & 95.05 & 0.800 & 0.83 \\
\hline 17 & 403 & 80 & 60 & 92.73 & 87.64 & 5.090 & 5.49 & 97.81 & 95.05 & 2.760 & 2.82 \\
\hline 18 & 373 & 40 & 30 & 51.03 & 44.25 & 6.780 & 13.29 & 65.81 & 63.9 & 1.910 & 2.90 \\
\hline 19 & 373 & 120 & 30 & 82.89 & 81.52 & 1.370 & 1.65 & 95.03 & 94.29 & 0.740 & 0.78 \\
\hline 20 & 403 & 80 & 60 & 92.07 & 87.64 & 4.430 & 4.81 & 98.67 & 95.05 & 3.620 & 3.67 \\
\hline
\end{tabular}

$A D$ absolute difference between experimental and predicted values 
runs. These results suggest that experimental factors affect removal percentages of each TOC and aniline in different ways. Moreover, harsher conditions are required to elevate TOC removal percentages when compared with the aniline removal percentages.

The absolute difference percentages $(\mathrm{AD}, \%)$ between experimental and theoretical TOC removal and aniline removal percentages are given in Table 2 . The AD percentage values are calculated by dividing the differences between the experimental and theoretical values by the experimental values and multiplying the results by 100 . Obtained results show that $\mathrm{AD}$ percentages remained minor in the all runs except run 2, runs 18 and 3, in the TOC removal model, and similarly, except for the run 5, the AD percentages were reasonable in the aniline removal model. Thus, it can be said that the experimental and predicted values are in accordance in both models.

\section{Evaluation of the CCD modeling}

Evaluation of the system variables and the relationship between these variables and responses were ensured using CCD modeling. Also, CCD was employed to obtain predicted values of both responses, namely TOC removal and aniline removal, for all runs using data given in Table 2. Predicted values were obtained using second-order polynomial equations (Eqs. 1 and 2) in terms of coded factors for both cases of TOC removal and aniline removal processes. Equations (1) and (2) can be used to predict any response of the system for given levels of system variables. Moreover, the effect of each variable can be identified by comparing its coefficient in the following equations:

$$
\begin{aligned}
Y_{1}= & 6.32 x_{1}+10.41 x_{2}+4.93 x_{3}+0.36 x_{1} x_{2}+3.94 x_{1} x_{3} \\
& -8.58 x_{2} x_{3}-11.53 x_{1}^{2}-5.00 x_{2}^{2}-0.91 x_{3}^{2}+87.64, \\
Y_{2}= & 2.85 x_{1}+7.55 x_{2}+4.98 x_{3}+0.83 x_{1} x_{2}+1.98 x_{1} x_{3} \\
& -8.48 x_{2} x_{3}-5.69 x_{1}^{2}-3.37 x_{2}^{2}-1.04 x_{3}^{2}+95.05 .
\end{aligned}
$$

In addition, the Pareto chart, which is functional to identify the relative impact of the variables, and facilitates the evaluation of the results, is displayed in Fig. 1 [19, 24]. This graphical analysis provides the demonstration of effects of each term in Eqs. (1) and (2). According to this figure, one can say that the quadratic effect of the temperature $\left(x_{1}^{2}\right)$ is the most effective factor on the TOC removal process, where the interaction effect of the $\mathrm{H}_{2} \mathrm{O}_{2}$ concentration and treatment time $\left(x_{2} x_{3}\right)$ is the most responsible factor for elevated yield in the case of aniline removal. $\mathrm{H}_{2} \mathrm{O}_{2}$ concentration $\left(x_{2}\right)$ is the second most effective factor in both cases of TOC and aniline removal. Moreover, the interaction effects of temperature and $\mathrm{H}_{2} \mathrm{O}_{2}$ concentration $\left(x_{1} x_{2}\right)$, and quadratic effect of treatment time $\left(x_{3}^{2}\right)$ were found to be negligible in both cases.

\section{ANOVA and regression coefficients of CCD models}

Experimental data were statistically analysed by ANOVA to evaluate the fit of the models. The results of ANOVA
Fig. 1 The Pareto chart of the process variables

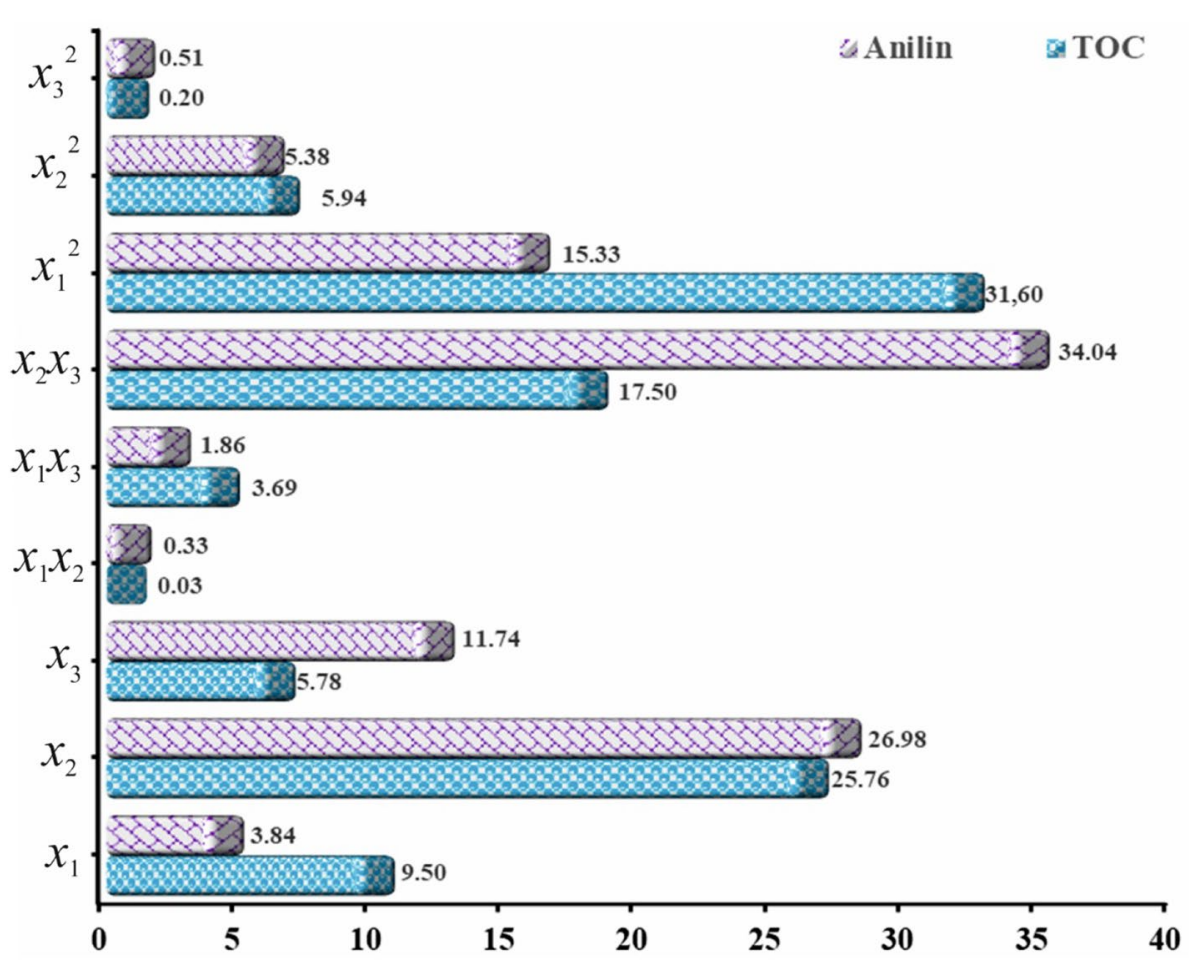


of the quadratic models, which were obtained by CCD, are demonstrated in Table 3. The statistical significancy of the applied models and models' terms can be expressed by ANOVA test factors such as $p$ and $F$ values [22]. $p$ and $F$ values of the TOC removal models were obtained as 12.70 and 0.0002 , respectively, and 9.76 and 0.0007 , respectively, for the aniline removal model. These values verify that both of the models can be used to confidently designate the effects of system variables on the response. 0.02 and 0.07 chances of $F$ values were obtained in the TOC removal and aniline removal, respectively. Model terms, which possess $p$ values less than 0.05 , are qualified as significant terms [21]. In this case, $x_{1}, x_{2}, x_{3}, x_{2} x_{3}, x_{1}^{2}$, and $x_{2}^{2}$ are significant terms of the TOC removal model and $x_{2}, x_{3}, x_{2} x_{3}, x_{1}^{2}$, and $x_{2}^{2}$ are significant terms of the aniline removal model.

Regression coefficients, which are demonstrated in Table 4, were used to evaluate the confidence of the CCD models. Lower values of the predicted residual sum of squares (PRESS) indicate the adaptation of points of the design to the model $[19,25]$. It was obtained that the aniline removal model better fits each point in the design than the TOC removal model according to the obtained PRESS values of the aniline removal model (782.53) and the TOC removal model (2613.93). Also, the conformity of the models can be presented by the coefficient of determination $\left(R^{2}\right)$ values, where higher values are desired $[19,25]$. Herein, the $0.9196 R^{2}$ value of the TOC removal model shows higher consistency between the system variables and the response than the aniline removal model considering its $R^{2}$ value of 0.8978 .

The standard deviation value of the aniline removal model (5.25) is more appropriate than the values of the TOC removal model (6.75). The percentage of standard deviation to the mean ratio, in other words, the coefficient of the variation value (CV \%) was obtained as 8.91 and 5.96 for the
Table 4 Regression coefficients of the CCD models

\begin{tabular}{lll}
\hline Regression coefficient & TOC removal & Aniline removal \\
\hline Standard deviation & 6.75 & 5.25 \\
Mean & 75.74 & 88.15 \\
CV \% & 8.91 & 5.96 \\
PRESS & 2613.93 & 782.53 \\
$R^{2}$ & 0.9196 & 0.8978 \\
Adjusted $R^{2}$ & 0.8472 & 0.8058 \\
Predicted $R^{2}$ & 0.5387 & 0.7100 \\
Adequate precision & 10.29 & 9.85 \\
\hline
\end{tabular}

TOC removal model and the aniline removal model, respectively. The signal-to-noise ratio value (adequate precision value) verifies the applicability of a model in the navigation of the design space and it is desired to be higher than 4 [26]. Adequate precision values of 10.29 and 9.85 , which were obtained for the TOC removal model and the aniline removal model, respectively, are in good agreement with the above-mentioned definition. Figure 2, in which the correlation between the actual and predicted values of (a) the TOC removal model and (b) the aniline removal model was displayed, supports this agreement. It is clearly seen from this figure that, actual and predicted values are in good accordance and these points verify good fit of both models.

Cube plots of (a) TOC removal model and (b) aniline removal model are displayed in Fig. 3. These figures make it easy to estimate the responses of the models for the combination of three independent factors between -1 and +1 levels. Also, they provide further analysis in the experimental region and estimation of experimental conditions for the desired response level. According to Fig. 3a, the highest TOC removal can be obtained as $87.58 \%$ at the highest level of all experimental variables. However, a quite reasonable
Table 3 ANOVA of the CCD models

\begin{tabular}{|c|c|c|c|c|c|c|}
\hline \multirow[t]{2}{*}{ Source } & \multicolumn{3}{|l|}{ TOC removal } & \multicolumn{3}{|c|}{ Aniline removal } \\
\hline & Mean square & $F$ value & $p$ value prob $>F$ & Mean square & $F$ value & $p$ value prob $>F$ \\
\hline Model & 578.98 & 12.70 & 0.0002 & 269.19 & 9.76 & 0.0007 \\
\hline$x_{1}$ & 545.16 & 11.96 & 0.0061 & 111.17 & 4.03 & 0.0724 \\
\hline$x_{2}$ & 1480.75 & 32.49 & 0.0002 & 778.01 & 28.21 & 0.0003 \\
\hline$x_{3}$ & 332.43 & 7.29 & 0.0223 & 339.26 & 12.30 & 0.0057 \\
\hline$x_{1} x_{2}$ & 1.05 & 0.023 & 0.8823 & 5.56 & 0.20 & 0.6629 \\
\hline$x_{1} x_{3}$ & 123.87 & 2.72 & 0.1303 & 31.32 & 1.14 & 0.3116 \\
\hline$x_{2} x_{3}$ & 589.62 & 12.94 & 0.0049 & 575.45 & 20.87 & 0.0010 \\
\hline$x_{1}^{2}$ & 1917.04 & 42.06 & $<0.0001$ & 467.01 & 16.94 & 0.0021 \\
\hline$x_{2}^{2}$ & 359.76 & 7.89 & 0.0185 & 163.30 & 5.92 & 0.0352 \\
\hline$x_{3}^{2}$ & 11.87 & 0.26 & 0.6209 & 15.69 & 0.57 & 0.4681 \\
\hline Residual & 45.58 & & & 27.57 & & \\
\hline Lack of fit & 63.14 & 2.25 & 0.1967 & 12.52 & 0.29 & 0.8976 \\
\hline Pure error & 28.02 & & & 42.63 & & \\
\hline
\end{tabular}



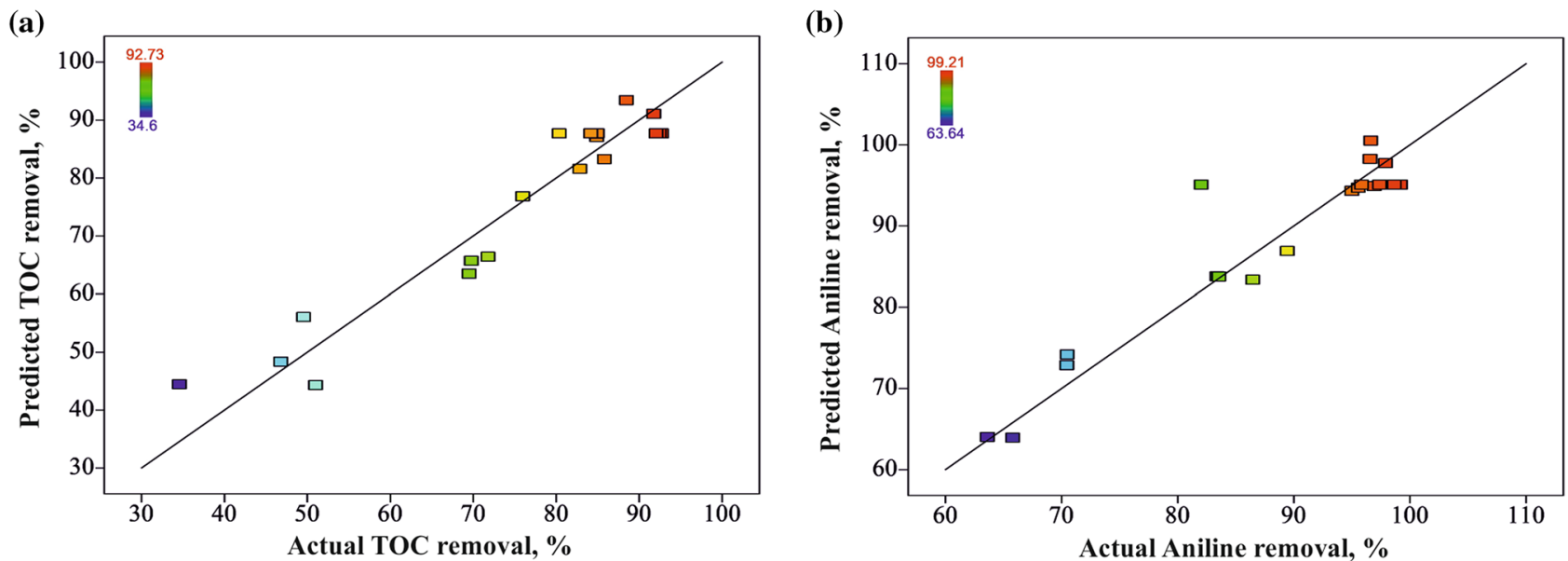

Fig. 2 Correlation between the actual and predicted values in a TOC removal model, $\mathbf{b}$ aniline removal model

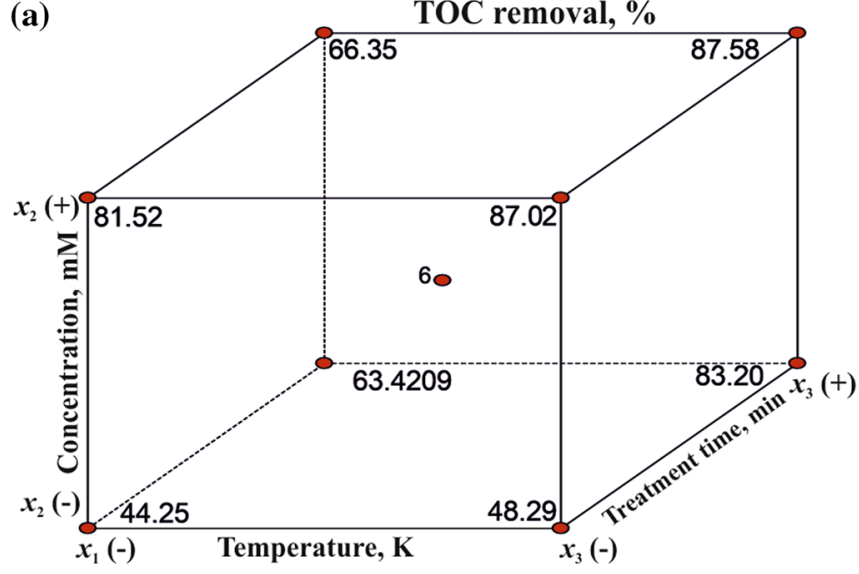

Fig. 3 The cube plot of a TOC removal model, $\mathbf{b}$ aniline removal model

value of $83.20 \%$ of the TOC removal can be obtained at the same conditions but at the lowest $\mathrm{H}_{2} \mathrm{O}_{2}$ concentration. Moreover, $81.52 \%$ of the TOC removal can be obtained at the highest level of $\mathrm{H}_{2} \mathrm{O}_{2}$ concentration and the lowest levels of temperature and treatment time. $94.29 \%$ of the aniline removal is an obtainable value at the highest $\mathrm{H}_{2} \mathrm{O}_{2}$ concentration level but at the lowest treatment time and the lowest temperature level. This efficiency can be increased up to 97.70 by increasing the last variable, temperature, to its highest level at the same conditions.

\section{Binary effects of the process variables on the removal yields}

Binary effects of the process variables on the response were displayed by three-dimensional plots. These schemes are utilized for presenting the interaction between process variables as well as for evaluating the optimum experimental

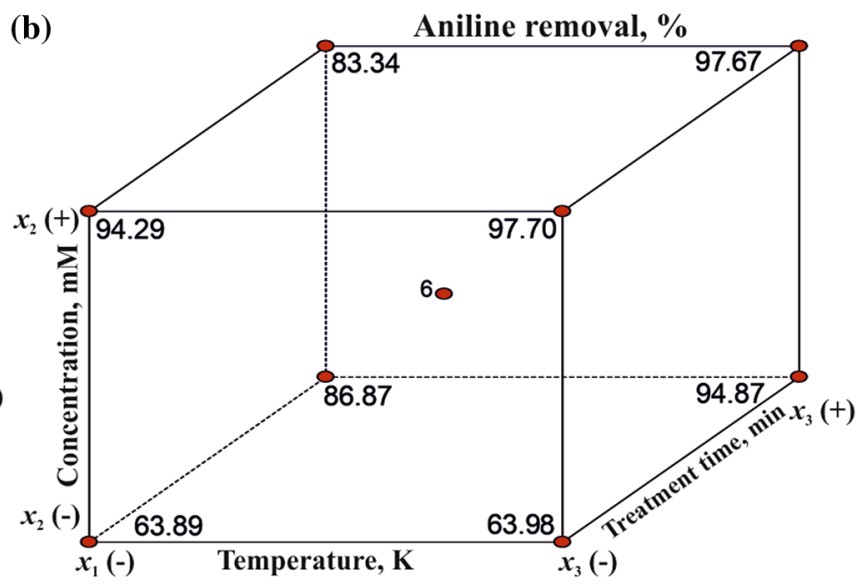

conditions for providing the maximum benefit of the response.

Binary effects of treatment time and $\mathrm{H}_{2} \mathrm{O}_{2}$ concentration on (a) TOC removal and (b) aniline removal yields at a fixed temperature of $420 \mathrm{~K}$ are demonstrated in Fig. 4. As seen from this figure, the interaction effect of treatment time and $\mathrm{H}_{2} \mathrm{O}_{2}$ concentration is crucial for both the TOC and the aniline removal. Namely, the red area, which presents a high-yielded area, was obtained at low $\mathrm{H}_{2} \mathrm{O}_{2}$ concentration and long treatment time or high $\mathrm{H}_{2} \mathrm{O}_{2}$ concentration and short treatment time at a fixed temperature of $420 \mathrm{~K}$. Moreover, elevated TOC removal and aniline removal percentages can be obtained at moderate levels of treatment time and $\mathrm{H}_{2} \mathrm{O}_{2}$ concentration. However, it should be noted that the obtained yield in the aniline removal, even at the lowest values of treatment time and $\mathrm{H}_{2} \mathrm{O}_{2}$ concentration, is higher than that of TOC removal. This can be explained by the fact that while the terms aniline removal stands for the 

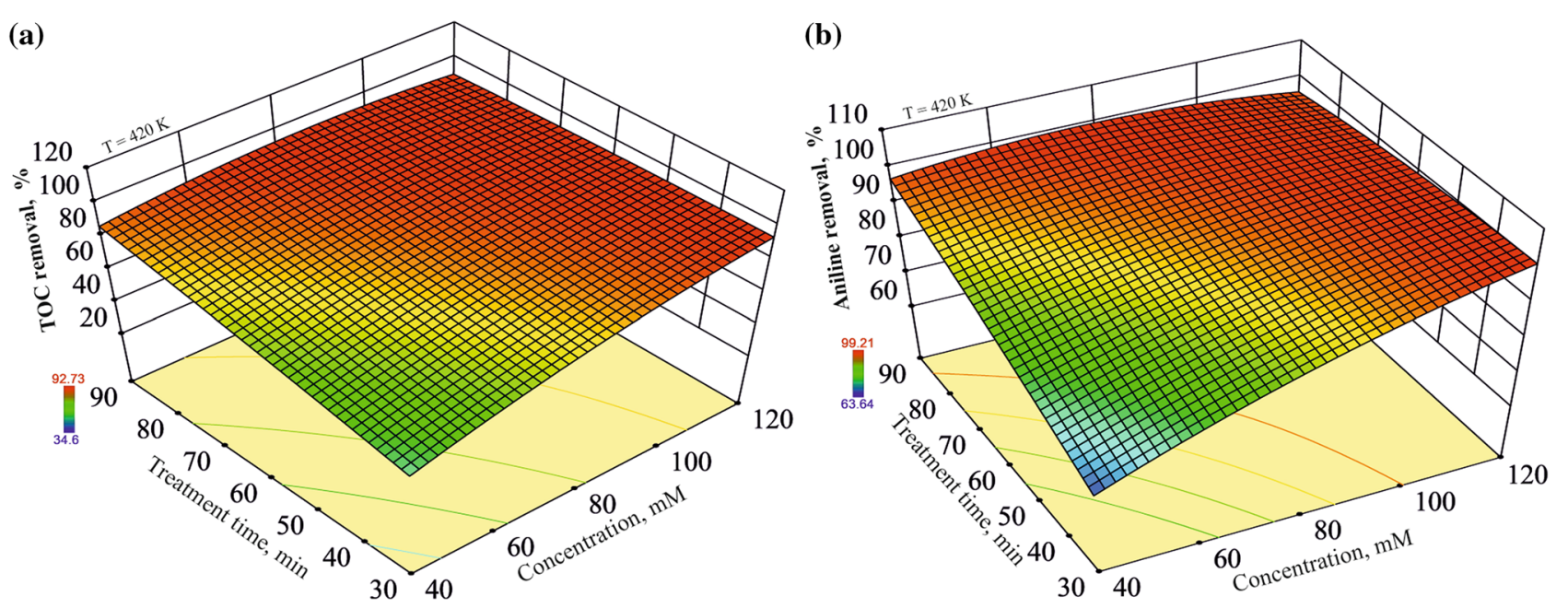

Fig. 4 Binary effects of treatment time and $\mathrm{H}_{2} \mathrm{O}_{2}$ concentration on a TOC removal and $\mathbf{b}$ aniline removal yields at a fixed temperature of $420 \mathrm{~K}$

UV spectrophotometric analysis and any bond break in the aniline structure or similar impact increase aniline removal percentages, the TOC removal percentage represents total mineralisation of aniline and harsher conditions are required for elevated TOC removal percentages.

Binary effects of temperature and $\mathrm{H}_{2} \mathrm{O}_{2}$ concentration on the TOC removal and aniline removal yields at a fixed treatment time of $30 \mathrm{~min}$ are shown in Fig. 5a, b, respectively. According to these figures, it is clear that the combined effects of temperature and $\mathrm{H}_{2} \mathrm{O}_{2}$ concentration are crucial on the TOC removal and aniline removal percentages. However, $\mathrm{H}_{2} \mathrm{O}_{2}$ concentration is the decisive factor for elevated yields in both cases. Increasing temperature from its lowest value to its highest value, under moderate levels of $\mathrm{H}_{2} \mathrm{O}_{2}$ concentration, has a minor effect on the TOC removal

(a)

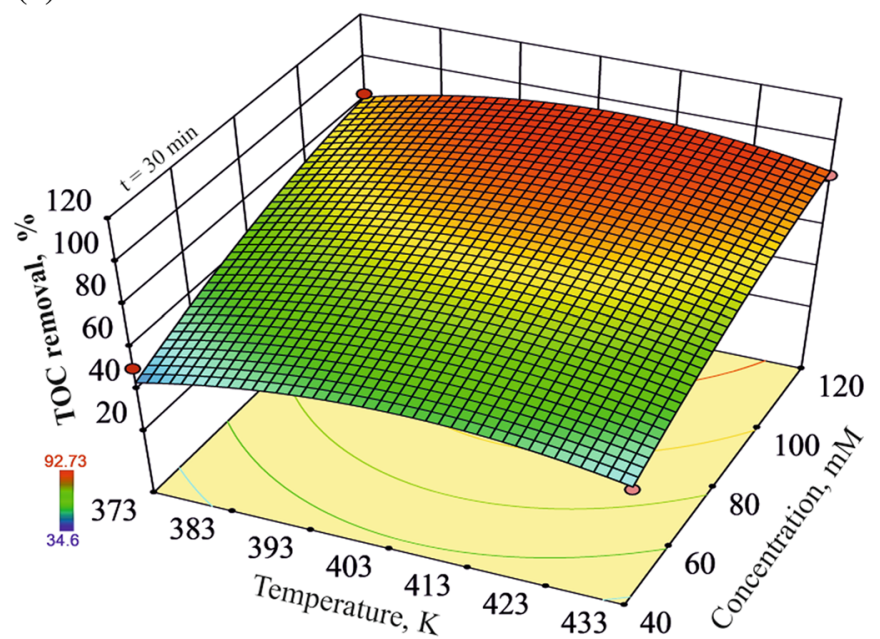

yields. However, increasing $\mathrm{H}_{2} \mathrm{O}_{2}$ concentration, from its moderate levels to its high levels, significantly increases the TOC removal yields above moderate levels of temperature. The fact that $\mathrm{H}_{2} \mathrm{O}_{2}$ concentration is a decisive factor appears more clearly in Fig. 5b. For instance, increasing temperature from 373 to $433 \mathrm{~K}$ at a fixed $\mathrm{H}_{2} \mathrm{O}_{2}$ concentration of $80 \mathrm{mM}$ and treatment time of $30 \mathrm{~min}$, provides an increase from 67.89 to $72.65 \%$ in the TOC removal, and from 67.89 to $72.65 \%$ in the aniline removal process. However, increasing $\mathrm{H}_{2} \mathrm{O}_{2}$ concentration from 80 to $120 \mathrm{mM}$ at a fixed temperature of $403 \mathrm{~K}$ and treatment time of $30 \mathrm{~min}$ provides a significant increase in the TOC removal (from 67.89 to $72.65 \%$ ) and the aniline removal (from 84.20 to $97.70 \%$ ) processes. This can be explained as follows: while the temperature is essential to activate $\mathrm{H}_{2} \mathrm{O}_{2}$ to hydroxyl (b)

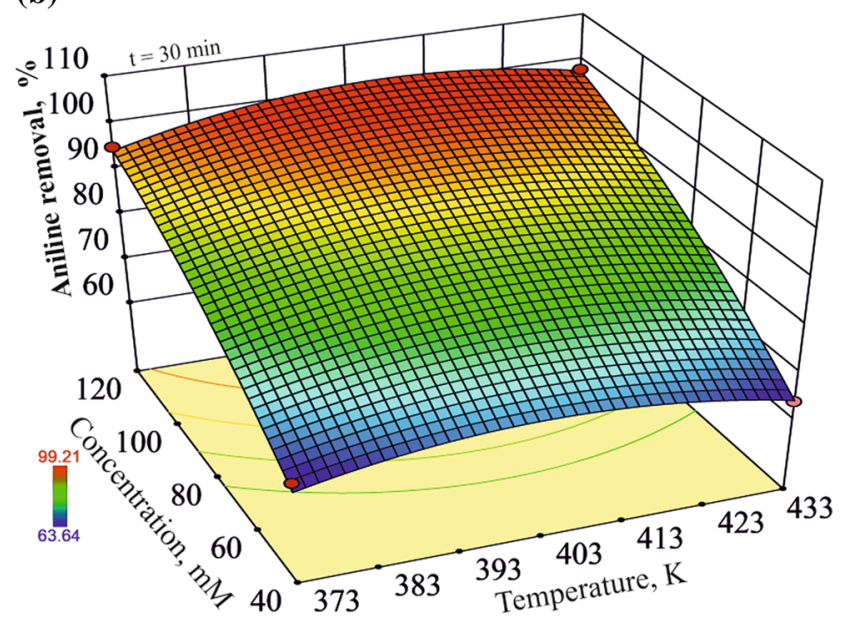

Fig. 5 Binary effects of temperature and $\mathrm{H}_{2} \mathrm{O}_{2}$ concentration on a TOC removal and $\mathbf{b}$ aniline removal yields at a fixed treatment time of 30 min 

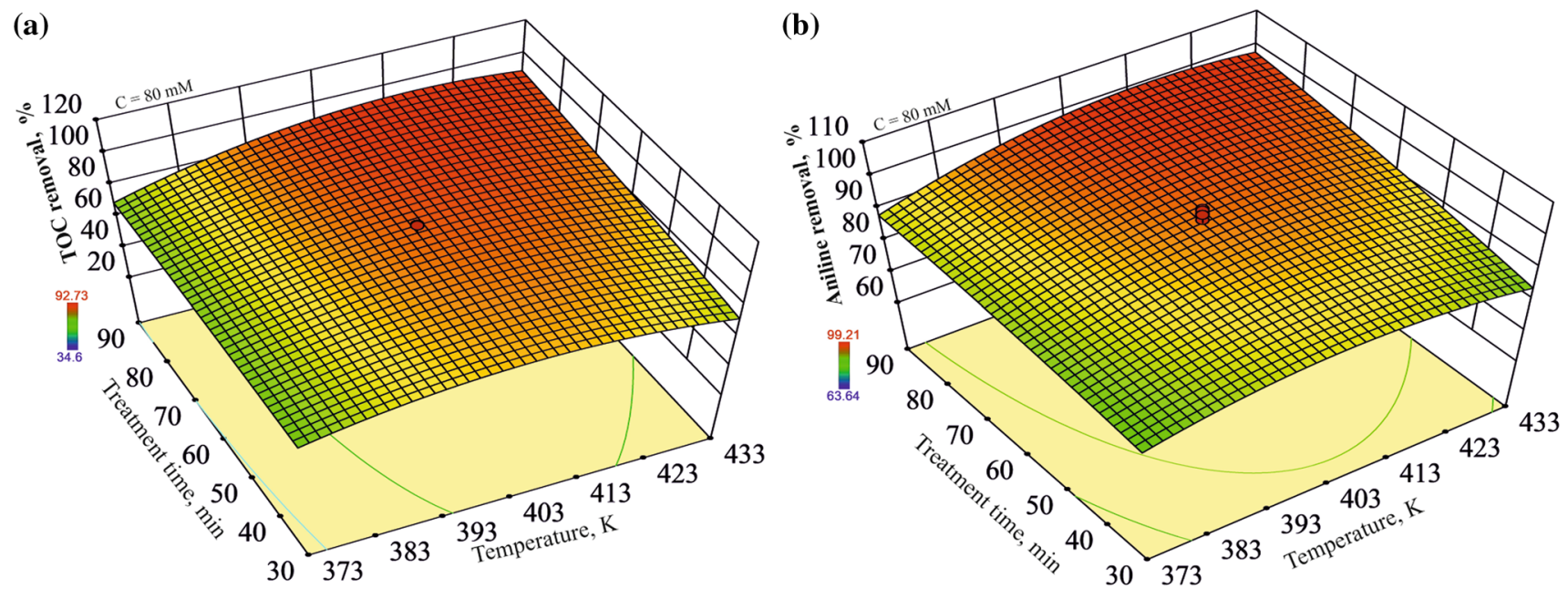

Fig. 6 Binary effects of treatment time and temperature on a TOC removal and $\mathbf{b}$ aniline removal yield at a fixed $\mathrm{H}_{2} \mathrm{O}_{2}$ concentration of $80 \mathrm{mM}$

radicals, more importantly, an adequate amount is required to obtain high efficiency in both cases of the TOC and the aniline removal.

The interaction of temperature and treatment time and their effects on the responses at a fixed $\mathrm{H}_{2} \mathrm{O}_{2}$ concentration of $80 \mathrm{mM}$ is shown in Fig. 6a, b. It is clearly seen in Fig. 6a that moderate TOC removal percentages can be obtained at moderate temperatures in all treatment times in the experimental region and at low temperature values. However, to obtain high yields, treatment time must be increased above its moderate levels, above $403 \mathrm{~K}$ of temperature. For instance, $81.80 \%$ of the TOC removal value increased to $91.67 \%$ by increasing treatment time from $30 \mathrm{~min}$ to $90 \mathrm{~min}$ at $80 \mathrm{mM}$ of $\mathrm{H}_{2} \mathrm{O}_{2}$ concentration and $403 \mathrm{~K}$ of temperature. According to Fig. 6b, the high-yielded area is limited to a specific region in the aniline removal process. Thus, the high yield can be obtained only above moderate levels of treatment time and temperature. These results suggest the fact that a sufficient amount of hydroxyl radicals may occur above specific values of temperature and treatment time, which are also dependent on the structure of the target molecules. $95.05 \%$ of aniline removal was obtained at the medium level of all variables, $60 \mathrm{~min}$ of treatment time, $80 \mathrm{mM}$ of $\mathrm{H}_{2} \mathrm{O}_{2}$ concentration and $403 \mathrm{~K}$ of temperature.

\section{Conclusion}

In this study, the aniline-contaminated aqueous solution, presenting a model of aniline-contaminated water sources, was purified by the synergistic effect of an environmentally friendly method, SWO and a green oxidizing agent, $\mathrm{H}_{2} \mathrm{O}_{2}$. Significant TOC and aniline removal percentages were obtained using the advantages of this work. It should be noted that this work is a good alternative to dysfunctional and ineffective conventional methods, according to achieved results. Moreover, CCD was used to comprehensively evaluate the TOC and aniline removal models and their variables, temperature, $\mathrm{H}_{2} \mathrm{O}_{2}$ concentration, and treatment time. Higher than $92 \%$ of TOC removal and $99 \%$ of aniline removal percentages were achieved. The applicability of the models and reliability of the model terms were evaluated with ANOVA.

Acknowledgements This work was funded by Mersin University Research Fund (Project no.: BAP 2017-2-AP5-2291) and linguistically supported by the Mersin Technology Transfer Office Academic Writing Center of Mersin University.

\section{Compliance with ethical standards}

Conflict of interest The authors declare that they have no conflict of interest.

Open Access This article is distributed under the terms of the Creative Commons Attribution 4.0 International License (http://creativeco mmons.org/licenses/by/4.0/), which permits unrestricted use, distribution, and reproduction in any medium, provided you give appropriate credit to the original author(s) and the source, provide a link to the Creative Commons license, and indicate if changes were made.

\section{References}

1. Orshansky F, Narkis N (1997) Characteristics of organics removal by pact simultaneous adsorption and biodegradation. Water Res 31:391-398. https://doi.org/10.1016/S0043-1354(96)00227-8

2. Zhang YQ, Xie XF, Huang WL, Huang SB (2013) Degradation of aniline by $\mathrm{Fe}^{2+}$-activated persulfate oxidation at ambient temperature. J Cent South Univ 20:1010-1014. https://doi.org/10.1007/ s11771-013-1577-9 
3. Sauleda R, Brillas E (2001) Mineralization of aniline and 4-chlorophenol in acidic solution by ozonation catalyzed with $\mathrm{Fe}^{2+}$ and UVA light. Appl Catal B 29:135-145. https://doi.org/10.1016/ S0926-3373(00)00197-1

4. Tang H, Li J, Bie Y, Zhu L, Zou J (2010) Photochemical removal of aniline in aqueous solutions: switching from photocatalytic degradation to photo-enhanced polymerization recovery. J Hazard Mater 175:977-984. https://doi.org/10.1016/j.jhazm at.2009.10.106

5. Matsushita M, Kuramitz H, Tanaka S (2005) Electrochemical oxidation for low concentration of aniline in neutral $\mathrm{pH}$ medium: application to the removal of aniline based on the electrochemical polymerization on a carbon fiber. Environ Sci Technol 39:38053810. https://doi.org/10.1021/es040379f

6. Canle LM, Santaballa JA, Vulliet E (2005) On the mechanism of $\mathrm{TiO}_{2}$-photocatalyzed degradation of aniline derivatives. J Photochem Photobiol A Chem 175:192-200. https://doi.org/10.1016/j. jphotochem.2005.05.001

7. Kamarehie B, Mohamadian J, Mousavi SA, Asgarid G, Shahamat YD (2017) Aniline degradation from aqueous solution using electro/ $\mathrm{Fe}^{2+} /$ peroxydisulphate process. Desalin Water Treat 80:337343. https://doi.org/10.5004/dwt.2017.20996

8. Bilal M, Rasheed T, Iqbal HMN, Li C, Wang H, Hu H, Wang W, Zhang X (2018) Photocatalytic degradation, toxicological assessment and degradation pathway of CI Reactive Blue 19 dye. Chem Eng Res Des 129:384-390. https://doi.org/10.1016/j.cherd .2017.11.040

9. Chen WS, Huang CP (2015) Mineralization of aniline in aqueous solution by electrochemical activation of persulfate. Chemosphere 125:175-178. https://doi.org/10.1016/j.chemosphere.2014.12.053

10. Wu X, Lei Z, Li Q, Zhu J, Chen B (2010) Liquid-liquid extraction of low-concentration aniline from aqueous solutions with salts. Ind Eng Chem Res 49:2581-2588. https://doi.org/10.1021/ie901 2979

11. Bilal M, Rasheed T, Iqbal HMN, Yan Y (2018) Peroxidasesassisted removal of environmentally-related hazardous pollutants with reference to the reaction mechanisms of industrial dyes. Sci Total Environ 644:1-13. https://doi.org/10.1016/j.scito tenv.2018.06.274

12. Laszlo K (2005) Adsorption from aqueous phenol and aniline solutions on activated carbons with different surface chemistry. Colloids Surfaces A Physicochem Eng Asp 265:32-39. https:// doi.org/10.1016/j.colsurfa.2004.11.051

13. Yabalak E, Gizir AM (2013) Subcritical and supercritical fluid extraction of heavy metals from sand and sewage sludge. J Serb Chem Soc 78:1013-1022. https://doi.org/10.2298/JSC1203211 $23 \mathrm{Y}$

14. Levec J, Pintar A (2007) Catalytic wet-air oxidation processes: a review. Catal Today 124:172-184. https://doi.org/10.1016/j.catto d.2007.03.035

15. Li L, Chen P, Gloyna EF (1991) Generalized kinetic model for wet oxidation of organic compounds. AIChE J 37:1687-1697. https:// doi.org/10.1002/aic.690371112
16. Klavariotia M, Mantzavinos D, Kassinos D (2009) Removal of residual pharmaceuticals from aqueous systems by advanced oxidation processes. Environ Int 35:402-417. https://doi. org/10.1016/j.envint.2008.07.009

17. Liotta LF, Gruttadauria M, Carlo GD, Perrini G, Librando V (2009) Heterogeneous catalytic degradation of phenolic substrates: catalysts activity. J Hazard Mater 162:588-606. https:// doi.org/10.1016/j.jhazmat.2008.05.115

18. Izadiyan P, Hemmateenejad B (2016) Multi-response optimization of factors affecting ultrasonic assisted extraction from Iranian basil using central composite design. Food Chem 190:864-870. https://doi.org/10.1016/j.foodchem.2015.06.036

19. Yabalak E (2018) Degradation of ticarcillin by subcritical water oxidation method: application of response surface methodology and artificial neural network modelling. J Environ Sci Heal A 11:975-985. https://doi.org/10.1080/10934529.2018.1471023

20. Manahan SE (2000) Environmental science, technology, and chemistry. CRC Press LLC, Boca Raton

21. Yabalak E (2017) Radical scavenging activity and chemical composition of methanolic extract from Arum dioscoridis sm. var. dioscoridis and determination of its mineral and trace elements. J Turk Chem Soc Sect Chem 5:205-218. https://doi.org/10.18596/ jotcsa. 350370

22. Yabalak E, Könen Adigüzel S, Adigüzel A, Ergene R, Tunçer M, Gizir AM (2017) Application of response surface methodology for the optimization of oxacillin degradation by subcritical water oxidation using $\mathrm{H}_{2} \mathrm{O}_{2}$ : genotoxicity and antimicrobial activity analysis of treated samples. Desalin Water Treat 81:186-198. https://doi.org/10.5004/dwt.2017.21089

23. Pilkington JL, Preston C, Gomes RL (2014) Comparison of response surface methodology (RSM) and artificial neural networks (ANN) towards efficient extraction of artemisinin from Artemisia апnиa. Ind Crops Prod 58:15-24. https://doi. org/10.1016/j.indcrop.2014.03.016

24. Hammami S, Oturan N, Bellakhal N, Dachraoui M, Oturan MA (2007) Oxidative degradation of direct orange 61 by electroFenton process using a carbon felt electrode: application of the experimental design methodology. J Electroanal Chem 610:75-84. https://doi.org/10.1016/j.jelechem.2007.07.004

25. Anderson MJ, Whitcomb PJ (2005) RSM simplified: optimizing processes using response surface methods for design of experiments. CRC Press, New York

26. Yabalak E (2018) An approach to apply eco-friendly subcritical water oxidation method in the mineralization of the antibiotic ampicillin. J Environ Chem Eng 6:7132-7137. https://doi. org/10.1016/j.jece.2018.10.010

Publisher's Note Springer Nature remains neutral with regard to jurisdictional claims in published maps and institutional affiliations. 